\title{
Quantified Car: Potenziale, Geschäftsmodelle und Digitale Ökosysteme
}

\author{
A. Stocker, C. Kaiser
}

Ein Fahrzeug ist ein „Hochleistungscomputer auf vier Rädern" und mit einer heterogenen Sensorik ausgestattet. Die von dieser Sensorik ermöglichte Sammlung von Fahrzeuglebenszyklusdaten erlaubt die Entwicklung völlig neuer Produkte, Services und Geschäftsmodelle. In den USA hat sich in diesem Kontext in Analogie zur Quantified-Self-Bewegung eine lebendige Quantified-Car-Startup-Szene etabliert, welche mit enormen Risikokapitalsummen von teilweise mehr als 20 Millionen USD ausgestattet ist. Diese Entwicklungen zeigen sehr deutlich, wie hoch der Marktwert eines Digitalen Ökosystems für Quantified Car durch Investoren eingeschätzt wird. Vor diesem Hintergrund liefert dieser Beitrag eine Einführung in das Phänomen Quantified Car und analysiert die Geschäftsmodelle der drei Startups Automatic, Mojio und Dash. Eine wesentliche Erkenntnis aus dieser Analyse besteht darin, dass sich die verfolgten Anwendungsszenarien, Dienste und Basistechnologien der Startups durchaus überdecken. Der Beitrag schließt mit einer kurzen Diskussion über den zunehmenden Wettbewerb zwischen der IKT-Industrie und den etablierten Automotive-Branchengrößen über die Vorherrschaft im Aufbau eines Digitalen Ökosystems für Quantified Car.

Schlüsselwörter: Quantified self; Quantified car; Digitale Ökosysteme; Geschäftsmodelle

\section{Quantified Car: potentials, business models and digital ecosystems.}

A modern vehicle is a "computer on four wheels" equipped with many different types of sensors. The so enabled continuous collection of vehicle lifecycle data facilitates the generation of innovative products, services and business models. In analogy to the QuantifiedSelf-movement, the USA has already evolved a plethora of Quantified Car startup companies backed by enormous amounts of risk capital, reaching more than 20 million USD in some cases. These developments clearly demonstrate how high investors perceive the market value of a working digital ecosystem for Quantified Car. This paper provides an introduction into the Quantified-Carphenomenon and analyses the business models of three different Quantified-Car-startups: Automatic, Mojio, and Dash. One major finding is that their use cases, services and underlying technologies show many similarities. The paper closes with a discussion on the increasing competition between the players from the ICT and the automotive domain on the supremacy in the development of a digital ecosystem for Quantified Car.

Keywords: Quantified Self; Quantified Car; digital ecosystems; business models

\section{Von Quantified Self zu Quantified Car}

In einer vernetzten Welt sammeln physische Dinge des täglichen Alltags immer mehr Daten über sich selbst und ihre Umgebung und transformieren langsam zu "Smart, Connected Products" [5, 6]. Produkte werden zur Datenquelle und "Data-Scientists" werten die über den gesamten Lebenszyklus dieser intelligenten Produkte gesammelten, stetig steigenden Datenmengen aus, um interessante Erkenntnisse über das Nutzer- bzw. das Produktverhalten zu gewinnen. Im Privatbereich nutzt etwa die Quantified-Self-Bewegung die Werkzeuge einer modernen Daten-gestützten Analyse dazu, vielschichtige Erkenntnisse über den eigenen Organismus zu gewinnen.

Als Begriff bezieht sich Quantified Self auf die wachsende Bereitwilligkeit vieler Menschen, Daten über sich, ihr Verhalten und ihre Umwelt zu sammeln, seien sie von biologischer, verhaltensorientierter, oder physikalischer Natur [9-11]. Heute wollen Millionen von "Quantified-Selfers" mit einer systematischen Datensammlung, -analyse und -auswertung vielschichtige Erkenntnisse zu persönlichen, gesundheitlichen, oder sportlichen Fragestellungen gewinnen. Häufig verwenden sie dazu ihr persönliches Smartphone, welches mit einer Vielzahl an Sensoren und Werkzeugen ausgestattet ist und den Quantified-Selfer täglich begleitet. Der bereits im Jahr $2007^{1}$ mit einem eigenen Begriff ausgezeichnete Quantified-Self-Trend zeigt seine wirtschaftliche Relevanz vor allem durch jüngste Akquisitionen von mobilen Smartphone Apps im Sport-Bereich. Etwa übernahm Adidas im Jahr 2015 für rund 220 Millionen Euro die Mehrheit am österreichischen Unternehmen Runtastic ${ }^{2}$. Das von Runtastic aufgebaute Ökosystem bietet mittlerweile für Konsumenten eine breite Palette an Gesundheits- und Fitnessprodukten, Services und Inhalten an. Die populäre App von Runtastic zählt mit Stand April 2016 be-

\footnotetext{
${ }^{1}$ What is The Quantified Self? www.quantifiedself.com/2011/03/what-is-thequantified-self, letzter Zugriff am 25.07.2016.

${ }^{2}$ Adidas Group acquires Runtastic: www.adidas-group.com/en/media/newsarchive/press-releases/2015/adidas-group-acquires-runtastic, letzter Zugriff am 25.07.2016
}

Stocker, Alexander, Virtual Vehicle Research Center, Inffeldgasse 21a, 8010 Graz, Österreich (E-Mail: alexander.stocker@v2c2.at); Kaiser, Christian, Virtual Vehicle Research Center, Inffeldgasse 21a, 8010 Graz, Österreich 
reits über 80 Millionen registrierte Nutzer ${ }^{3}$. Dieses Beispiel zeigt sehr deutlich, dass etablierte Branchengrößen mittlerweile hohe Summen investieren, um sich an innovativen Technologie-Startups im Quantified-Self-Umfeld zu beteiligen. Der Analyst Gartner rechnet gar mit einem Marktvolumen von 5 Milliarden USD für QuantifiedSelf-Geräte für das Jahr 2016 [4].

Im Leben vieler Menschen sind Fahrzeuge eine große Anschaffung und mit hohem Wert und Interesse für ihre Besitzer verbunden Das Interesse an durch eine Quantifizierung von Fahrzeuglebenszyklusdaten gewonnenen Erkenntnissen, wie beispielsweise Objektzustand diagnostizieren, Fahrstil analysieren und optimieren, oder durch Integration von Umgebungsdaten die eigene Fahrsicherheit verbessern, kann vor allem bei Fahrzeug-Enthusiasten als besonders hoch eingeschätzt werden. Denn auch Fahrzeuge besitzen ein hohes Potenzial, als Datenquelle neue Services zu ermöglichen. Bezeichnet der Begriff Quantified Self die Aufzeichnung, Analyse und Auswertung der im Rahmen eigener Aktivitäten erzeugten Daten, umfasst der Begriff Quantified Car in Analogie dazu das systematische Sammeln der Lebenszyklusdaten eines Fahrzeugs in der Nutzungsphase und darauffolgend die intelligente Analyse, um für unterschiedliche Stakeholder einen Nutzen zu stiften.

\section{Quantified Car als Enabler von Digitalen Ökosystemen}

Aufgrund ständiger Veränderungen im Wettbewerbsumfeld durch die digitale Transformation herrscht ein hoher Anpassungsdruck bei den Unternehmen. Im Zentrum der aktuellen Digitalisierungsdiskussion steht vor allem die Vernetzung von technischen Geräten, die im Alltag genutzt werden [12]. Digitale Ökosysteme stellen den Lebensraum von digitalen Inhalten dar, indem sie ein technisch abgegrenztes System bilden, das Hardware, Software, Inhalte und Services miteinander vernetzt [1]. Nach dieser Systematik besteht beispielsweise das vielzitierte Apple-Ökosystem aus iPod, iPhone, iPad, Mac Desktop, MacBooks und Peripheriegeräten (Hardware), iOS/Mac OS, Office Suite \& Core Apps, iTunes \& iBooks, Tools für Media Developer (Software), Musik Podcasts, Hörbücher, MusikVideo, TV-Serien, Filme, eBooks, iBooks, Textbooks (Inhalte), iCloud und iTunes (Dienste). Die durch solche Digitale Ökosysteme entstehenden Chancen werden durch Risiken minimiert, nicht zuletzt deshalb, weil Anbieter stetig damit konfrontiert sind, ihre Leistungen sowie die zugrunde liegenden Geschäftsmodelle zu überdenken, um bei Notwendigkeit strategische Maßnahmen zu ergreifen, die eine klassische, innovative oder disruptive Neu- bzw. Umorientierung bewirken [3]

Auch rund um den Quantified-Self-Trend haben sich mittlerweile Digitale Ökosysteme gebildet: So sind neben klassischen Smartphones eine Reihe an smarten Produkten wie Smart Glasses, Smart Watches, oder Fitness-Armbänder verfügbar, um weitere Facetten einer kontinuierlichen Datensammlung und Datenanalyse zu ermöglichen. Aus diesem Grund bieten die Marktplätze von Google und Apple eine unüberschaubare Anzahl an Quantified-SelfApplikationen an. Einige Hersteller der oben angeführten Smart, Connected Products wie beispielsweise das von Intel akquirierte Unternehmen Recon Instruments ${ }^{4}$ bieten bereits eigene Marktplätze, auf denen auch Dritte Anwendungen bereitstellen können.

Im Vergleich zu Quantified Self befindet sich die Quantified-CarBewegung noch am Anfang, und die Nutzerzahlen sind entsprechend geringer. Es wird heute im Hinblick auf die Entwicklung von

\footnotetext{
${ }^{3}$ www.runtastic.com/mediacenter/corporate-assets/german/companyoverview/20160405_corporate_overview_de.pdf, letzter Zugriff am 25.07.2016.

${ }^{4}$ Recon Instruments: www.reconinstruments.com, letzter Zugriff am 25.07.2016.
}

Fahrerassistenzsystemen intensiv über die Vernetzung des Fahrzeugs

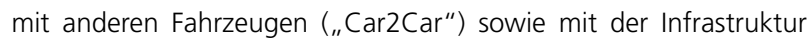
("Car2X") diskutiert. Doch eine innovative Verwertung der von Fahrzeugen gesammelten Lebenszyklusdaten mit Fokus auf die Generierung von Mehrwert für den Fahrenden - in Analogie zur Quantified-Self-Bewegung - hat bei den europäischen Fahrzeugherstellern noch keinen hohen Stellenwert erreicht. Damit bleibt die Frage bisher unbeantwortet, wie das Quantified-Self-Phänomen erfolgreich auf das Fahrzeug übertragen werden kann.

Moderne Fahrzeuge sind Hochleistungscomputer auf vier Rädern. Ausgestattet mit einer umfangreichen und vielschichtigen Sensorik sammeln sie bereits eine Unmenge an Daten über sich selbst und mit der breiten Verfügbarkeit von Fahrerassistenzsystemen auch immer mehr über ihre Umgebung. Laut dem von Volkswagen koordinierten EU-Projekt AutoMat ${ }^{5}$, welches sich mit technischen Fragestellungen rund um die Etablierung eines Marktplatzes für Fahrzeuglebenszyklusdaten befasst, verarbeitet ein modernes Fahrzeug pro Sekunde bis zu 4000 Signale im in Fahrzeugen etablierten Controller Area Network (CAN)-Bus System ${ }^{6}$ und stellt damit einen viel umfassenderen und interessanteren Sensor-Messknoten und Datengenerator dar, als die von Quantified-Selfern zur Datensammlung genutzten Smartphones und Wearables. Die kontinuierliche Sammlung und laufende Analyse von Fahrzeugdaten dient heute einem zentralen Zweck, der Gewährleistung bzw. Überwachung von Fahrzeugfunktionen. Doch die Möglichkeiten einer weiterführenden, intelligenten Nutzung dieser Daten gehen weit über diesen originären Zweck hinaus, denn es ließen sich völlig neue Produkte, Dienstleistungen und sogar Digitale Ökosysteme entwickeln.

\section{Quantified Car-Ökosysteme}

\subsection{Akteure eines Digitalen Ökosystems für Quantified Car} In einem Quantified-Car-Ökosystem können folgende Akteure definiert werden:

- Primäre Endnutzer sind als individuelle Servicekonsumenten Fahrer/Eigentümer von Fahrzeugen, die direkt und unmittelbar aus innovativen Produkten, Visualisierungen, Statistiken, GamificationElementen und Fahrstiloptimierungs-Empfehlungen profitieren, deren Grundlage sie mit dem Bereitstellen ihrer Daten geschaffen haben.

- Sekundäre Endnutzer sind Organisationen bzw. Organisationseinheiten wie beispielsweise Stadtplaner, Versicherungen oder Flottenbetreiber, welche indirekt einen Nutzen durch gesammelte und ausgewertete Fahrzeuglebenszyklusdaten ziehen, indem sie die durch Serviceprovider bereitgestellten Dienstleistungen konsumieren.

- Serviceprovider sind wiederum Organisationen, welche Produkte/Services für primäre und/oder sekundäre Endnutzer anbieten und dabei Umsätze lukrieren. Dazu zählen bspw. Flottenmanagement-Serviceprovider, Serviceprovider für fahrstilabhängige Versicherungen, oder Serviceprovider für Services zur präventiven Fahrzeugwartung. Alle Services basieren auf Daten, die von primären Endnutzern bereitgestellt werden.

- Cloudprovider (Plattformbetreiber) sind dafür verantwortlich, die gesamte Infrastruktur eines Digitalen Ökosystems zu betreiben

${ }^{5}$ Automotive Big Data Marketplace for Innovative Cross-sectorial Vehicle Data Services: http://www.automat-project.eu/content/about-automa, letzter Zugriff am 25.07.2016.

${ }^{6}$ ISO 11898-1:2015 „Road vehicles_Controller area network (CAN)—Part 1: Data link layer and physical signaling". 


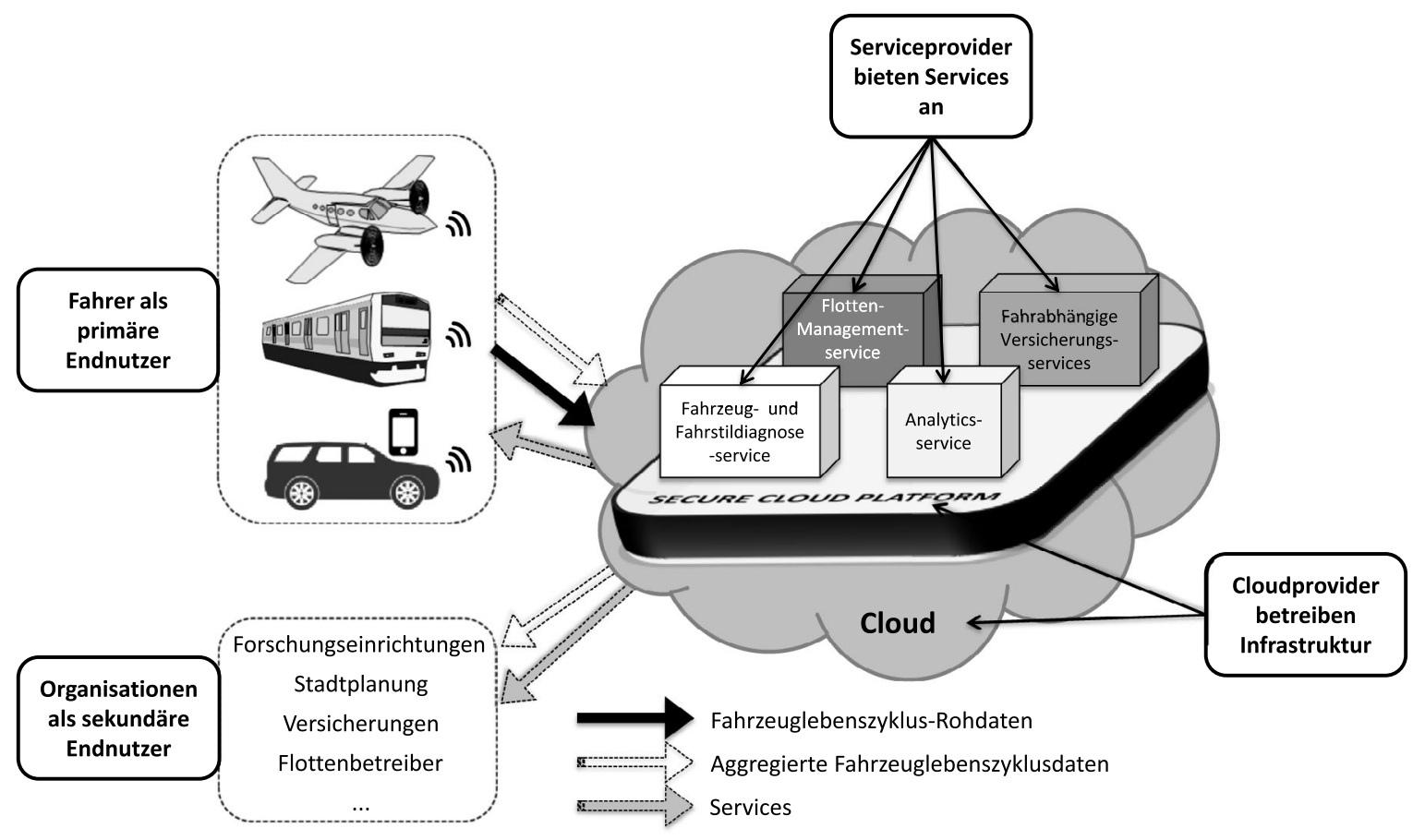

Abb. 1. Akteure eines Digitalen Ökosystems für Quantified Car

und für Serviceprovider zur Verfügung zu stellen. Primäre und sekundäre Endnutzer sind als individuelle bzw. organisationale Servicekonsumenten Nutzer von den durch Serviceprovider in dieser Cloud-Infrastruktur bereitgestellten Services (Fig. 1).

\subsection{Innovationstreiber USA - quo vadis Europa?}

Während in den USA seit einigen Jahren enorme Anstrengungen unternommen werden, um Digitale Ökosysteme für Quantified Car zu erschließen, geht diese Entwicklung an Europa fast völlig vorbei. Abseits des bereits erwähnten EU-Projekts AutoMat finden in Europa nur vereinzelte und vergleichsweise kleine Aktivitäten ohne signifikanten Impact statt. Dem gegenüber hat sich in den USA eine sehr lebendige, mit enormen Risikokapitalinvestitionen von teilweise mehr als USD 20 Mio. finanzierte, Startup-Szene etabliert, wie nachfolgende Tab. 1 darstellt. Neben der IT-Szene investieren laut CrunchBase, einem Portal mit Informationen über innovative Technologieunternehmen und dazugehörige Investoreninformationen, auch Unternehmen wie Magna International, Continental ITS, und BMW i Ventures.

Diese Entwicklungen zeigen sehr anschaulich, dass auch die Automotive-Branche den Marktwert eines Digitalen Ökosystems für Quantified Car als enorm einschätzt, wenngleich sie dort aus heutiger Sicht nicht den Innovationstreiber darstellt. Das von Volkswagen koordinierte EU-Projekt Automat führt drei wesentliche Gründe $\mathrm{an}^{7}$, warum gerade die Automotive-Industrie mit ihren ConnectedBestrebungen heute noch nicht in der Lage ist, ein offenes und umfassendes Digitales Ökosystem zu etablieren:

- Derzeit sind Angebote rund um das Connected Vehicle durch markenspezifische Geschäftsansätze geprägt, welche in proprietäre und geschlossene Einzellösungen gemündet sind. Original Equipment Manufacturers (OEMs) treten als Fahrzeughersteller in völlig

${ }^{7}$ Automat-Projekt: www.automat-project.eu/content/objectives, letzter Zugriff am 25.07.2016. neue Märkte ein, die nicht notwendigerweise mit ihrem Kerngeschäft korrelieren.

- Aktuelle Connected-Services fokussieren auf den individuellen Fahrzeugkäufer, was unweigerlich zu Datenschutzbedenken führt. Derzeit wird nicht berücksichtigt, wie anonymisierte Fahrzeuglebenszyklusdaten in anderen Kontexten überhaupt genutzt werden könnten, die nicht den individuellen Fahrer betreffen.

- Das assoziierte Risiko einer Zusammenarbeit zwischen den im Wettbewerb befindlichen OEMs hinsichtlich einer gemeinsamen, standardisierten Bereitstellung von Fahrzeuglebenszyklusdaten in einem Digitalen Ökosystem ist eine wesentliche Hürde, warum sich ein solches noch nicht etabliert hat.

\section{Fallbeispiele für Quantified-Car-Ökosysteme}

Nachfolgend werden die Geschäftsmodelle der drei Quantified-CarFallbeispiele Automatic, Mojio und Dash nach der Methodik von Stähler [7] analysiert, welche Geschäftsmodelle grob nach den drei Aspekten Nutzenversprechen, Architektur der Wertschöpfung und Ertragsmodell differenziert.

\subsection{Quantified-Car-Fallbeispiel 1: Automatic}

Nutzenversprechen: Nach dem Motto "Connect your car to the rest of your digital life" bietet das Unternehmen Automatic $\operatorname{Labs}^{8}$ aus San Francisco Applikationen für Endkunden und Business-Nutzer an. Um diese nutzen zu können, muss in einem ersten Schritt ein spezieller Adapter an der Standarddiagnoseschnittstelle (OBD) eines Fahrzeuges betrieben werden, welcher Fahrzeugdaten über ein via Bluetooth gepaartes Smartphone unterschiedlichen Apps als Datenbasis zur Verfügung stellt, die über einen eigenen Marktplatz, die Automatic Gallery, bezogen werden können.

${ }^{8}$ Automatic Labs: www.automatic.com, letzter Zugriff am 25.07.2016 
Tab. 1. Quantified-Car-Startups in den USA ${ }^{a}$

\begin{tabular}{|c|c|c|c|c|c|}
\hline Unternehmen & Nutzenversprechen & Verwendungsmöglichkeiten & Geschäftsanwendungen & Kosten & Investoren \\
\hline $\begin{array}{l}\text { automatic.com } \\
\text { San Francisco } \\
\text { (USA), } \\
\text { gegründet } \\
2011\end{array}$ & $\begin{array}{l}\text { Verbindet Auto mit } \\
\text { dem digitalen Leben } \\
\text { des Fahrers. Befähigt } \\
\text { Fahrer mit Wissen über } \\
\text { sich selbst und das } \\
\text { Fahrzeug darin, } \\
\text { sicherer und smarter zu } \\
\text { fahren. }\end{array}$ & $\begin{array}{l}\text { Verbindet das Fahrzeug mit } \\
\text { vielen Apps, etwa für } \\
\text { Problemdiagnose, } \\
\text { Verbrauchsoptimierung, } \\
\text { Standort- und } \\
\text { Notfallservices. Beinhaltet } \\
\text { ein Web- dashboard mit } \\
\text { umfangreichen Statistiken. }\end{array}$ & $\begin{array}{l}\text { Cloud-basierte Services } \\
\text { für Versicherungsunter- } \\
\text { nehmen, } \\
\text { Flottenbetreiber und } \\
\text { Fahrzeughersteller, } \\
\text { Bereistellug der Daten- } \\
\text { analyseinfrastruktur. }\end{array}$ & $\begin{array}{l}\$ 99,95 \text { für den } \\
\text { OBD2-Adapter zum } \\
\text { Einstieg in das } \\
\text { digitale Ökosystem, } \\
\text { keine Informationen } \\
\text { zu den Preisen der } \\
\text { BusinessServices }\end{array}$ & $\begin{array}{l}\$ 24 \text { Mio in } 3 \\
\text { Runden von } 13 \\
\text { Investoren }\end{array}$ \\
\hline $\begin{array}{l}\text { moj.io } \\
\text { Vancouver } \\
\text { (CAN), } \\
\text { gegründet } \\
\text { 10/2012 }\end{array}$ & $\begin{array}{l}\text { Empowerment des } \\
\text { Fahrers. Verbinde dich } \\
\text { jederzeit mit deinen } \\
\text { Devices mit deinem } \\
\text { Fahrzeug. }\end{array}$ & $\begin{array}{l}\text { Zugriff auf einen } \\
\text { Marktplatz für Apps zur } \\
\text { Fahrzeug-Vernetzung } \\
\text { (Standorttracking, } \\
\text { Fahrzeugdiagnose, } \\
\text { FahrAnalysen,..), offene } \\
\text { Plattform zum Zugriff auf } \\
\text { das Fahrzeug, } \\
\text { DeveloperCenter, API. }\end{array}$ & Nicht explizit genannt & $\begin{array}{l}\text { \$149 für den } \\
\text { OBD2-Adapter zum } \\
\text { Einstieg in das } \\
\text { digitale Ökosystem. } \\
\text { Inkludiert 3G/4G } \\
\text { Konnektivität. }\end{array}$ & $\begin{array}{l}\text { \$10.3 Mio in } 3 \\
\text { Runden von } 6 \\
\text { Investoren }\end{array}$ \\
\hline $\begin{array}{l}\text { dash.by New } \\
\text { York (USA), } \\
\text { gegründet } \\
06 / 2012\end{array}$ & $\begin{array}{l}\text { Smartes, sicheres, } \\
\text { grünes und leistbares } \\
\text { Fahren ermöglichen. }\end{array}$ & $\begin{array}{l}\text { Connected Car Plattform, } \\
\text { Dienste zur } \\
\text { Fahrzeugdiagnose, } \\
\text { Verbrauchseffizienz, } \\
\text { Notfalldienste, } \\
\text { Fahrervergleiche, } \\
\text { Gamification und } \\
\text { Community-Dienste. }\end{array}$ & Nicht explizit genannt & $\begin{array}{l}\text { Funktioniert auch } \\
\text { mit OBD2- } \\
\text { Adaptern anderer } \\
\text { Hersteller, die u.a. } \\
\text { im Webshop von } \\
\text { dash bezogen } \\
\text { werden können }\end{array}$ & $\begin{array}{l}\$ 1.9 \text { Mio in } 3 \\
\text { Runden von } 8 \\
\text { Investoren }\end{array}$ \\
\hline $\begin{array}{l}\text { vin.li Dallas } \\
\text { (USA), } \\
\text { gegründet } \\
2014\end{array}$ & $\begin{array}{l}\text { Dein Fahrzeug - dein } \\
\text { Weg. Bringt unzählige } \\
\text { Apps in das Fahrzeug, } \\
\text { von Sicherheit über } \\
\text { Entertainment bis zu } \\
\text { WIFI. }\end{array}$ & $\begin{array}{l}\text { Hochgeschwindigkeits- } \\
\text { WIFI, App-Store für das } \\
\text { Fahrzeug, alle Arten von } \\
\text { smarten Fahrzeug-Apps } \\
\text { und Services zum } \\
\text { Download in einem } \\
\text { eigenen AppStore, } \\
\text { Developer-Portal. }\end{array}$ & Nicht explizit genannt & $\begin{array}{l}\text { \$199.99 für den } \\
\text { OBD2-Adapter zum } \\
\text { Einstieg in das } \\
\text { Digitale Ökosystem. } \\
\text { Inkludiert 3G/4G } \\
\text { Konnektivität. } \\
\text { Zusätzliche Kosten } \\
\text { für Internet/Wifi je } \\
\text { nach gewünschtem } \\
\text { Datenvolumen. }\end{array}$ & $\begin{array}{l}\$ 6.5 \text { Mio in } 2 \\
\text { Runden von } 6 \\
\text { Investoren }\end{array}$ \\
\hline $\begin{array}{l}\text { zendrive.com } \\
\text { San Francisco } \\
\text { (USA), } \\
\text { gegründet } \\
2013\end{array}$ & $\begin{array}{l}\text { Sichere Fahrer - sichere } \\
\text { Straßen, } \\
\text { Smartphone-gestützte } \\
\text { Sicherheit auf Straßen } \\
\text { in Städten, für Flotten } \\
\text { und Individuen. }\end{array}$ & $\begin{array}{l}\text { Analysen von Smartphone- } \\
\text { Sensordaten durch } \\
\text { Machine learning. Fahrer- } \\
\text { und Flottenanalysen. } \\
\text { Kollisionserkennung, Versi- } \\
\text { cherungsUnterstützung, } \\
\text { etc. Developer-Kit und API. }\end{array}$ & $\begin{array}{l}\text { Flottenservices, } \\
\text { Versicherungen, } \\
\text { Car-sharing, }\end{array}$ & $\begin{array}{l}\text { Unterschiedliche } \\
\text { Preismodelle, von } \\
\text { kostenlos bis } \$ 4 \text { pro } \\
\text { Fahrer/Monat für } \\
\text { Flotten. } \\
\text { Businesstarife sind } \\
\text { nicht angeführt. }\end{array}$ & $\begin{array}{l}\$ 15 \text { Mio in } 3 \\
\text { Runden von } 15 \\
\text { Investoren }\end{array}$ \\
\hline $\begin{array}{l}\text { zubie.com } \\
\text { Sullivans Island } \\
\text { (USA), } \\
\text { gegründet } \\
05 / 2012\end{array}$ & $\begin{array}{l}\text { Wir machen das Fahren } \\
\text { sicherer, einfacher \& } \\
\text { günstiger. Verbindet } \\
\text { dein Fahrzeug mit dem } \\
\text { Internet, um } \\
\text { Echtzeit-Informationen } \\
\text { am Smartphone zu } \\
\text { erhalten. }\end{array}$ & $\begin{array}{l}\text { Fahr-Erkenntnisse, } \\
\text { VerhaltensWarnungen, } \\
\text { Ranglisten, Wartungs } \\
\text { Hinweise, Motordiagnose, } \\
\text { Batteriewarnung, } \\
\text { Notfallservices, Tracking, } \\
\text { Bewegungsaufzeichnung, } \\
\text { Vergünstigungen und } \\
\text { Tankstellen finden. }\end{array}$ & $\begin{array}{l}\text { Lösung für } \\
\text { Versicherungen und } \\
\text { Fahrzeug-Anbieter, } \\
\text { Flottentracking, GPS } \\
\text { tracking, FahrzeugZu- } \\
\text { standsüberwachung, } \\
\text { Fahrerverhalten. }\end{array}$ & $\begin{array}{l}\text { Unterschiedliche } \\
\text { Preismodelle, von } \\
\text { einmalig } \$ 99 \text { für } \\
\text { OBD2-Adapter und } \\
\$ 10 \text { pro Monat. } \\
\text { Business Nutzung } \\
\text { ab } \$ 17,95 \text { pro } \\
\text { Monat. }\end{array}$ & $\begin{array}{l}\$ 25,87 \text { Mio in } \\
5 \text { Runden von } \\
8 \text { Investoren }\end{array}$ \\
\hline
\end{tabular}

a Risikokapital und Investment-Informationen von Crunchbase.com: www.crunchbase.com, letzter Zugriff am 25.07.2016. 
Architektur der Wertschöpfung: Automatic bietet seit 2011 eine Reihe von Services für private Nutzer an. Dazu gehören beispielsweise umfangreiche Statistiken zu Fahrten am Smartphone sowie in einem Browser-Dashboard, Funktionen zur Diagnose von Motor- und Steuergeräteproblemen, Feedback zum jeweiligen Fahrstil, Funktionen zum Wiederauffinden eines geparkten Fahrzeugs, Kollisionsdetektionen mit Notfallservices, bis hin zur Möglichkeit mittels IFTTT ${ }^{9}$ (If This, Then That) Fahrzeugfunktionen über Automatic mit anderen digitalen Services aus dem Web zu verknüpfen. Automatic Labs bietet auch Services für Business Kunden an. Dazu zählen etwa der Betrieb einer Automotive Cloud, Cloud-basierte Fahrzeugversicherungen, Services für die intelligente Wartung von Fahrzeugen sowie für eine erhöhte Kundenbindung im After-Sales, Flottenmanagement sowie Datenanalysen. Spezifische Lösungen für OEMs sind in Planung.

Ertragsmodell: Der Automatic OBD-II Adapter ist in den USA für 99,95 USD erhältlich und für den Einstieg in das Quantified-CarÖkosystem von Automatic nötig (Lock-in-Effekt). Alle Services sind auf die USA begrenzt. Preise für Business-Lösungen werden auf der Website von Automatic Labs nicht aktiv kommuniziert.

\subsection{Quantified-Car-Fallbeispiel 2: Mojio}

Nutzenversprechen: Ähnlich wie Automatic möchte auch Mojio ${ }^{10}$ den Fahrer "empowern", wenn er sich mit einem einzigen Device, dem Smartphone, jederzeit mit seinem Fahrzeug verbinden kann. Auch bei Mojio besteht die Schnittstelle zwischen Smartphone und Fahrzeug in einem am OBD-II Port des Fahrzeuges betriebenen Adapter.

Architektur der Wertschöpfung: Auch Mojio bietet unterschiedlichste Apps und Services für Fahrer an. Diese reichen über Location Tracking, Fahrzeugdiagnose, Fahr-Analytics, Fahrstilanalyse und umfassen auch die Nutzung von mobilen Apps, die durch Dritte im Digitalen Ökosystem von Mojio bereitgestellt werden. Mit dem Developer Center bietet Mojio auch eine offene Connected-Car Plattform an, die über Application Programming Interfaces (APIs) und Software Development Kits (SDKs) verfügt, damit auch Dritte möglichst einfach Apps entwickeln können.

Ertragsmodell: Mojio betreibt einen Online Shop, in dem auch der OBD-II-Adapter inklusive einer eingebauten Sim-Karte für das AT\&T Mobilfunknetz um USD 149 in den USA bezogen werden kann. Damit besteht bei Mojio eine direkte Konnektivität des Fahrzeugs zum Internet - und nicht wie bei Automatic Labs nur über ein gepaartes Smartphone.

\subsection{Quantified-Car-Fallbeispiel 3: Dash}

Nutzenversprechen: Gemäß dem Motto "Smarter.Driving.Everyday." werden mit der App des 2012 gegründeten New Yorker Unternehmens Dash ${ }^{11}$ mit Hilfe eines OBD-II-Adapters erneut Fahrzeuglebenszyklusdaten gesammelt, um Fahrer in Echtzeit über interessante Ereignisse zu informieren. In der Business Variante werden Fahrzeuglebenszyklusdaten in aggregierter Form auch für Flotten dargestellt.

Architektur der Wertschöpfung: Die Dash-App ist für Android und IOS frei erhältlich und kann mit mehreren am Markt verfügbaren OBD-II-Adaptern kommunizieren, welche etwa über den Online-Shop von Dash bezogen werden können. Highlights von Dash sind Analysen von Fahrverhalten und Fahrzeugzustand, Bewertungen und Ranglisten (Community-Funktionen), persönliche

\footnotetext{
${ }^{9}$ IFTTT: ifttt.com, letzter Zugriff am 25.07.2016.

${ }^{10}$ Mojio: www.moj.io, letzter Zugriff am 25.07.2016.

${ }^{11}$ Dash: www.dash.by, letzter Zugriff am 25.07.2016.
}

Trend-Analysen sowie eine Kartenfunktion, um das geparkte Fahrzeug wiederzufinden. Auch Dash bietet mit der Dash Chassis API unter dem Schlagwort "Internet of Cars" eine Plattform für Entwickler an.

Ertragsmodell: Von TechCrunch, einem populären Online-Nachrichtenportal für Technologie- und Internet-Unternehmen, als „FitBit for Cars" bezeichnet, will Dash aus den gesammelten Fahrzeuglebenszyklusdaten über eine eigene Analytics-Plattform („Dash IQ“) interessante Erkenntnisse generieren, welche auch anderen Organisationen angeboten werden. Dash ist auch Projektpartner des Projekts DriveSmart ${ }^{12}$ des New York City Department of Transportation $^{13}$, bei dem es darum geht, dass Fahrer durch das Feedback aus der App Zeit- und Geld sparen und dabei noch sicherer fahren. Beispielsweise bekommen Fahrer eine Belohnung, wenn sie in New York außerhalb der Rush-Hour fahren oder weniger überlastete Strecken nutzen. Informationen zu konkreten Erlösquellen sind auf der Website allerdings nicht ersichtlich.

\section{Zusammenfassung und Diskussion}

Nach einer Einführung in das Quantified-Car-Phänomen und in das Bestreben von Startups aus den USA, Digitale Ökosysteme in diesem Bereich zu etablieren, wurden die Geschäftsmodelle der Quantified-Car-Fallbeispiele, Automatic, Mojio und Dash nach der Methodik von Stähler [7] beschrieben. Dabei ist auffallend, dass alle drei Player ähnliche Anwendungsszenarien verfolgen. Sie zielen jeweils darauf ab, aus den während der Nutzungsphase gesammelten Fahrzeuglebenszyklusdaten relevante Informationen zu erzeugen und diese für Fahrer entsprechend zu visualisieren. Am weitesten verbreitet scheint, gemäß einer Analyse der Downloadzahlen von Android-Installationen auf Google Play, Dash (100.000-500.000 Downloads) vor Automatic (10.000-50.000 Downloads) und Mojio (1.000-5.000 Downloads) zu sein. Nachfolgende Abbildung zeigt exemplarische Screenshots der jeweiligen Basis-Apps, wodurch sich die Ähnlichkeiten der Anwendungsszenarien auch aus der Gestaltung der Benutzeroberfläche erschließen lassen können (Abb. 2).

Zusammengefasst ist festzuhalten, dass der Kraftstoff für erfolgreiche Quantified-Car-Ökosysteme die durch Fahrer bereitgestellten Fahrzeuglebenszyklusdaten sind. Nur wenn eine kritische Masse an Fahrer eine kritische Masse an Daten freiwillig bereitstellt, können diese Digitalen Ökosysteme überhaupt erst entstehen. Doch dazu bedarf es vielfältiger Anreize, welche vermutlich nur durch interessante und kostenlose Services mit Mehrwert für Fahrer generiert werden können.

Die Henne-Ei Problematik zeigt sich darin, dass Fahrer eine große Menge an Daten bereitstellen, damit Dritte überhaupt Anwendungen auf Basis dieser entwickeln, doch viele Fahrer werden vermutlich überhaupt erst dann Daten bereitstellen, wenn interessante und nutzenstiftende Services bereits existieren. Vom Henne-Ei-Problem des "Web of Cars" können Parallelen zum Henne-Ei-Problems des Web of Data gezogen werden $[2,8]$. Um eine solche Henne-EiProblematik überhaupt überwinden zu können, sind die im Beitrag recherchierten Startups zum Teil mit sehr einfachen Anwendungen gestartet, welche Fahrende rasch einen Nutzen wahrnehmen lassen sollen.

Im Vergleich zur bereits etablierten Quantified-Self-Bewegung befindet sich die Quantified-Car-Bewegung gerade in Europa noch am Beginn. Das Thema Datenschutz ist hier vor allem im deutschsprachigen Raum in Politik, Gesellschaft und Industrie sehr stark

\footnotetext{
${ }^{12}$ Drive Smart: www.drivesmartnyc.com, letzter Zugriff am 25.07.2016.

${ }^{13}$ New York City DOT: www.nyc.gov/html/dot/html/home/home.shtml, letzter Zugriff am 25.07.2016.
} 

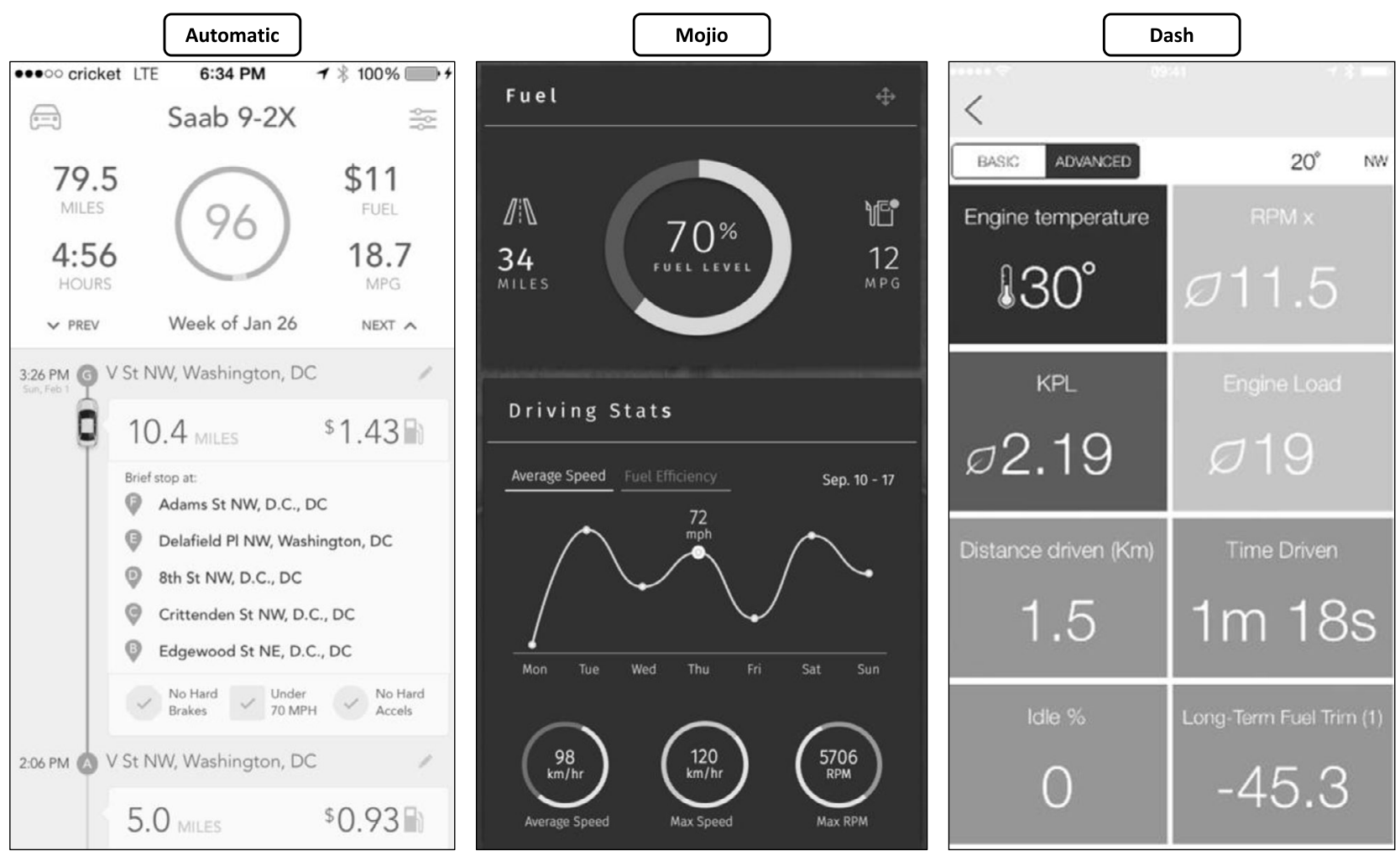

Abb. 2. Exemplarische Screenshots der GUls von Quantified-Car-Apps

verankert. Projekte mit Bezug zu datenschutzrelevanten Aspekten müssen dementsprechend mit einer sehr sensiblen Vorgehensweise durchgeführt werden. Nicht nur deswegen ist davon auszugehen, dass eine radikale datengetriebene-Innovation im Umfeld Quantified Car nicht unbedingt durch die europäischen Fahrzeughersteller aus Sicht der Technologieentwicklung stattfinden wird.

Dennoch sind bereits erste Aktivitäten zur Datensammlung und -übertragung im Zusammenhang mit neuen Fahrzeugen in Europa passiert, wie etwa ein Artikel von Heise Online als Berichterstattung zu einem ADAC-Experiment beschreibt. ${ }^{14}$ Aus diesen Aktivitäten wird aber weder ersichtlich, wie gesammelte Fahrzeuglebenszyklusdaten einen Nutzen für den Fahrer generieren sollen, noch wie Fahrer durch selektive Datenschutz-Einstellungen eine partielle oder totale Datenweitergabe konfigurieren bzw. verhindern können.

Gerade in einem solchen sensiblen Umfeld preschen Startups aus den USA, die nicht unter einer "Privacy-Bürde" leiden, mit frischen Ideen vor. Vergleichbar mit den Aktivitäten von IT-Größen wie Google, Apple und Facebook wird das Thema Datenschutz "erstmal etwas" in den Hintergrund gerückt. Der Wettkampf zwischen IT-Unternehmen und etablierten Branchengrößen aus der Automotive-Industrie um die Vorherrschaft bei der Etablierung Digitaler Ökosysteme rund um Fahrzeuglebenszyklusdaten wird sicherlich spannend, wie auch ein aktuelles Diskussionspapier des BVDW beschreibt ${ }^{15}$. Mit Sicherheit besteht die langfristige Verwer-

\footnotetext{
${ }^{14}$ ADAC-Untersuchung: Autohersteller sammeln Daten in großem Stil: www. heise.de/newsticker/meldung/ADAC-Untersuchung-Autohersteller-sammelnDaten-in-grossem-Stil-3227102.html, letzter Zugriff am 25.07.2016.

${ }^{15}$ Connected Cars - ein Diskussionspapier zum Thema Services des BVDW - Bundesverband Digitale Wirtschaft: www.bvdw.org/presseserver/Connected Cars/Finalversion_Diskussionspapier_Services_15.06.pdf, letzter Zugriff am 25.07.2016.
}

tungsstrategie einiger Quantified-Car-Startups darin, Technologie samt Nutzerbasis an Branchengrößen aus der Automotive oder ITIndustrie zu verkaufen. Eine solche Strategie lassen schon die sehr hohen Risikokapitalinvestitionen auf der einen Seite und die intransparenten Erlösmodelle der Startups auf der anderen Seite erahnen.

Abschließend soll an dieser Stelle explizit darauf hingewiesen werden, dass auch in Europa in den letzten Jahren eine Reihe von Startups mit dem Fokus auf die Entwicklung von Diensten rund um die Themen Mobilität und Smart Cities entstanden sind. Diese verfügen jedoch über weitaus weniger Risikokapital als ihre "Konkurrenten" aus den USA und sind damit langfristig vermutlich nicht konkurrenzfähig. Etwa findet sich in Österreich mit Parkbob ${ }^{16}$ auch ein innovativer Hersteller einer Smart Parking Anwendung, welcher im Jahr 2016 ein Investment von 250.000 EUR lukriert hat. Die Relevanz des Themas Smart Parking haben auch schon deutsche Fahrzeughersteller wahrgenommen. So arbeitet etwa der deutsche Premiumhersteller BMW im Rahmen der ConnectedDrive-Initiative gemeinsam mit dem US-Unternehmen und Verkehrsdatenanalysten INRIX ${ }^{17}$ (143 Mio. USD in 7 Runden von 6 Investoren laut Crunchbase.com) an einer entsprechenden Lösung für intelligentes Parken

\section{Danksagung}

Diese Arbeit entstand am VIRTUAL VEHICLE Research Center in Graz, Österreich. Die Autoren bedanken sich für die Förderung im Rahmen des COMET K2 - Competence Centers for Excellent Technologies Programms des Österreichischen Bundesministeriums

\footnotetext{
${ }^{16}$ Parkbob: www.parkbob.com, letzter Zugriff am 25.07.2016

${ }^{17}$ INRIX: www.inrix.com, letzter Zugriff am 25.07.2016.
} 
für Verkehr, Innovation und Technologie (bmvit), des Österreichischen Bundesministeriums für Wissenschaft, Forschung und Wirtschaft (bmwfw), der Österreichischen Forschungsförderungsgesellschaft $\mathrm{mbH}$ (FFG), des Landes Steiermark sowie der Steirischen Wirtschaftsförderung (SFG)

Open Access This article is distributed under the terms of the Creative Commons Attribution 4.0 International License (http://creativecommons.org/ licenses/by/4.0/), which permits unrestricted use, distribution, and reproduction in any medium, provided you give appropriate credit to the origina author(s) and the source, provide a link to the Creative Commons license, and indicate if changes were made.

\section{Literatur}

1. Ammon, T., Brem, A. (2013): Digitale Ökosysteme und deren Geschäftsmodelle: Analyse und Implikationen für klassische Buchverlage. In Keuper, F. et al. (Hrsg.) Digitalisierung und Innovation: Entwicklungsperspektiven, Planung, Entstehung (S. 93-121). Wiesbaden: Springer Gabler.

2. Latif, A., US Saeed, A., Höfler, P., Stocker, A., Wagner, C. (2009): The linked data value chain: a lightweight model for business engineers. In Proceedings of I-SEMANTICS (S. 568-575).

\section{Autoren}

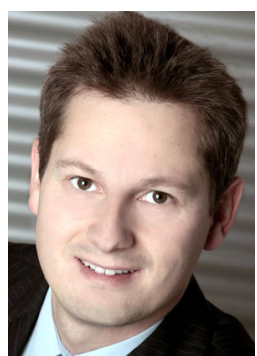

\section{Alexander Stocker}

beschäftigt sich seit über 14 Jahren in Wissenschaft und Praxis mit dem Einsatz computergestützter Informationssysteme in Unternehmen. Derzeit arbeitet er als Key Researcher für Information \& Process Management am Kompetenzzentrum - Das Virtuelle Fahrzeug in Graz, Österreich. Zuvor war er Key Researcher am Institut DIGITAL bei Joanneum Research, Executive Assistant to the CEO am Know-Center und Berater für Informationsmanagement und Informationstechnologie bei Datev.
3. Matt, C., Hess, T., Benlian, A. (2015): Digital transformation strategies. Bus. Inf. Syst. Eng., 57(5), 339-343. 2015.

4. McIntyre, A. (2013): Market trends: enter the wearable electronics market with products for the quantified self. Gartner report 2013. https://www.gartner.com/doc 2537715/market-trends-enter-wearable-electronics. Letzter Zugriff am 25.07.2016.

5. Porter, M. E., Heppelmann, J. E. (2014): How smart, connected products are transforming competition. In Harvard Business Manager.

6. Porter, M. E., Heppelmann, J. E. (2015): How smart, connected products are transforming companies. In Harvard Business Manager.

7. Stähler, P. (2002): Geschäftsmodelle in der digitalen Ökonomie. 2. Aufl. Lohmar: EUL Verlag.

8. Stocker, A., Tochtermann, K., Scheir, P. (2010): Die Wertschöpfungskette der Daten Eine Basis für zukünftige wirtschaftswissenschaftliche Betrachtungen des Web of Data. HMD, Prax. Wirtsch.inform., 47(5), 94-104.

9. Swan, M. (2009): Emerging patient-driven health care models: an examination of health social networks, consumer personalized medicine and quantified self-tracking. Int. J. Environ. Res. Public Health, 2009(6), 492-525.

10. Swan, M. (2013): The quantified self: fundamental disruption in big data science and biological discovery. Big Data, 1(2), 85-99. 2013.

11. Swan, M. (2015): Connected car: quantified self becomes quantified car. J. Sens. Actuator Netw., 4(1), 2-29. 2015.

12. Yoo, Y., Henfridson, O., Lyytinen, K. (2010): The new organizing logic of digital innovation: an agenda for information systems research. Inf. Syst. Res., 21(4), 724-735.

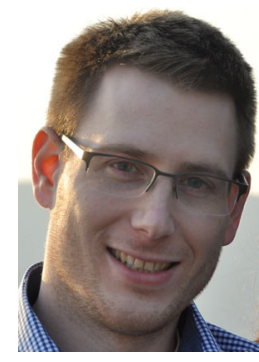

\section{Christian Kaiser}

beschäftigt sich als Senior Researcher am Kompetenzzentrum - Das Virtuelle Fahrzeug mit Konzeption, Implementierung und Evaluierung von anwenderorientiertem Design in Mensch-Maschine Kommunikation von betrieblichen Informationssystemen in der Automobilindustrie-Entwicklung. Insbesondere interaktive Web-Visualisierungen von betrieblichen Daten in Verbindung mit semantischen Technologien wurden von ihm im Rahmen von Forschungsprojekten mit der Automobilindustrie als Demonstratoren eigenständig konzipiert, umgesetzt und evaluiert. 